\title{
Detection and Evaluation of Pre-Preg Gaps and Overlaps in Glare Laminates
}

\author{
Davide $\operatorname{Nardi}^{1}$ (D) Morteza Abouhamzeh ${ }^{1} \cdot$ \\ Rob Leonard ${ }^{2} \cdot$ Jos Sinke $^{1}$
}

Received: 6 February 2018 / Accepted: 22 February 2018 / Published online: 13 March 2018

(C) The Author(s) 2018

\begin{abstract}
Gaps and overlaps between pre-preg plies represent common flaws in composite materials that can be introduced easily in an automated fibre placement manufacturing process and are potentially detrimental for the mechanical performances of the final laminates. Whereas gaps and overlaps have been addressed for full composite material, the topic has not been extended to a hybrid composite material such as Glare, a member of the family of Fibre Metal Laminates (FMLs). In this paper/research, the manufacturing, the detection, and the optical evaluation of intraply gaps and overlaps in Glare laminates are investigated. As part of an initial assessment study on the effect of gaps and overlaps on Glare, only the most critical lay-up has been considered. The experimental investigation started with the manufacturing of specimens having gaps and overlaps with different widths, followed by a nondestructive ultrasonic-inspection. An optical evaluation of the gaps and overlaps was performed by means of microscope image analysis of the cross sections of the specimens. The results from the non-destructive evaluations show the effectiveness of the ultrasonic detection of gaps and overlaps both in position, shape, width, and severity. The optical inspections confirm the accuracy of the non-destructive evaluation also adding useful insights about the geometrical features due to the presence of gaps and overlaps in the final Glare laminates. All the results justify the need for a further investigation on the effect of gaps and overlaps on the mechanical properties.
\end{abstract}

Keywords Fibre metal laminates · Gaps and overlaps · Ultrasonic non-destructive evaluation · Optical inspection · Defect onset

Davide Nardi

d.nardi@tudelft.nl

1 Faculty of Aerospace Engineering, Delft University of Technology, Delft 2629 HS, The Netherlands

2 Fibre Metal Laminates Centre of Competence, Kluyverweg 1, 2629 HS Delft, The Netherlands 


\section{Introduction}

Fibre Metal Laminates (FMLs) are hybrid materials consisting of alternating layers of metal sheets and composite layers [1]. Among them, Glare is the best-known member, consisting of aluminium layers and glass fibre reinforced composite layers, see Fig. 1.

Glare has six different standard grades. All of them are based on pre-preg, made of unidirectional S-glass fibres embedded in a FM 94 adhesive. This pre-preg can be laid-up according to different orientations with respect to the aluminium alloy sheet, resulting in the different grades [3]. Table 1 presents the current Glare grades along with the most significant material advantages. A proper laminate coding system is used in order to uniquely define laminates from Table 1.

An example is the following: Glare $2 \mathrm{~A}-3 / 2-0.4$, defined as

- Glare laminate with fibre orientation according to the Glare 2A definition in Table 1;

- 3 layers of aluminium and 2 composite layers; each composite layer consists of two pre-preg layers,

- Each aluminium layer is $0.4 \mathrm{~mm}$ thick.

Glare, as a hybrid, combines some of the advantages of either the aluminium alloy or the composite material. Examples are: better impact properties, damage tolerance and toughness when compared to composites, and better fatigue resistance, corrosion resistance, lower specific weight and ability for tailoring, when compared to aluminium alloys [3, 4].

Nonetheless, Glare is not immune for the presence of flaws or defects, which could basically be related to both composite and metal physical features. For clarity of notation, a flaw is here intended as a deviation from a perfect material, while a defect is considered as a deviation outside an agreed specification, mainly based on the effect on the mechanical properties of the considered component.

After the manufacturing process, flaws and defects can be found in the cured laminates, namely delaminations, voids, porosity, inclusions, disbonds, and cracks in the aluminium layers, which deteriorate the mechanical properties of the structural component. Hence an accurate and comprehensive understanding of the manufacturing process is essential in order to prevent the onset and evolution of flaws or defects.

Non-Destructive Evaluation (NDE) are inspection routines that allow the assessment of the structural health of the material to reduce manufacturing costs and out of service time of the aircraft due to maintenance operations. However, NDE methods for Glare materials represent a challenging

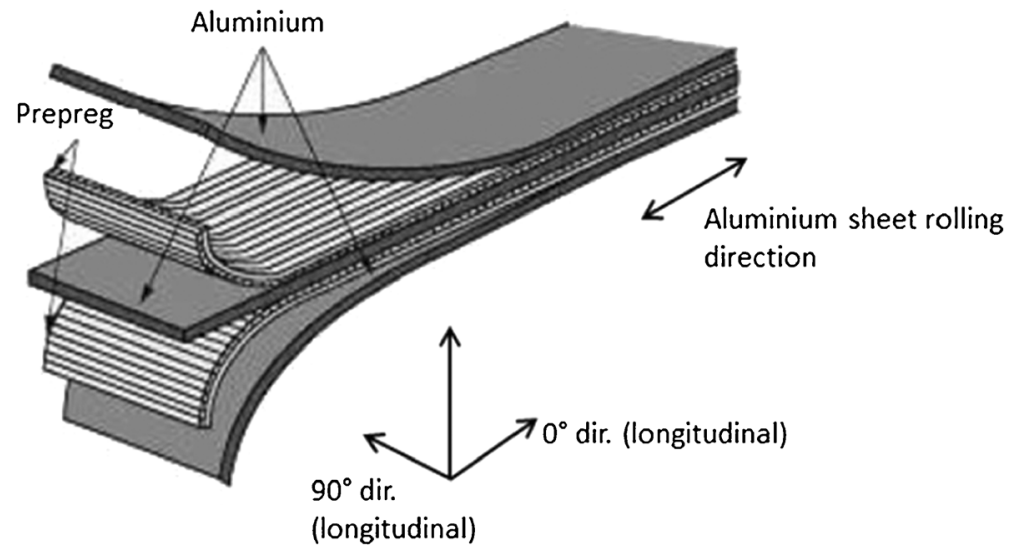

Fig. 1 Typical laminate lay-up for Glare [2] 
Table 1 Standard Glare grade [3]

\begin{tabular}{lllll}
\hline Glare grade & sub & $\begin{array}{l}\text { Metal sheet } \\
\text { thickness [mm] \& alloy }\end{array}$ & $\begin{array}{l}\text { Pre-preg orientation } \\
\text { in each fibre layer }\end{array}$ & Main advantages \\
\hline Glare 1 & - & $0.3-0.47575-\mathrm{T} 761$ & $0 / 0$ & fatigue, strength, yield stress \\
Glare 2 & Glare 2A & $0.2-0.52024-\mathrm{T} 3$ & $0 / 0$ & fatigue, strength \\
& Glare 2B & $0.2-0.52024-\mathrm{T} 3$ & $90 / 90$ & fatigue, strength \\
Glare 3 & - & $0.2-0.52024-\mathrm{T} 3$ & $0 / 90$ & fatigue, impact \\
Glare 4 & Glare 4A & $0.2-0.52024-\mathrm{T} 3$ & $0 / 90 / 0$ & fatigue, strength in $0^{\circ}$ direction \\
& Glare 4B & $0.2-0.52024-\mathrm{T} 3$ & $90 / 0 / 90$ & fatigue, strength in $90^{\circ}$ direction \\
Glare 5 & - & $0.2-0.52024-\mathrm{T} 3$ & $0 / 90 / 90 / 0$ & sheact \\
Glare 6 & Glare 6A & $0.2-0.52024-\mathrm{T} 3$ & $+45 /-45$ & shear, off-axis properties \\
& Glare 6B & $0.2-0.52024-\mathrm{T} 3$ & $+45 /-45$ &
\end{tabular}

topic due to Glare's highly anisotropic nature and the different properties of each ply which can make the detection difficult.

Various approaches have been considered for the assessment of the quality of Glare structures. They can be grouped in three main techniques: Eddy Current, Ultrasonic, and Infrared Thermography. Most of the time, they were also considered in the same work for comparison purposes.

Fahr et al. [5] determined the extent of damage caused by impact, corrosion and fatigue loads in Glare material by means of Air-Coupled Ultrasonic and Eddy Current methods. Heida and Platenkamp [6] also considered the Eddy Current technique, and Ultrasonic Techniques for the in-service inspection of sub-surface cracks at fastener rows in Glare fuselage structures. Sinke [7] presented a brief overview in which the quality control and the in-service inspection of Glare panels are addressed. The C-Scan method proves to be an effective tool for the quality control, while the Eddy Current method results to be one of the suitable options for in-service inspection. In a further study, Heida et al. [8] performed an investigation on the capability of the Eddy Current sliding probe technique for the in-service detection of cracks in Glare structures, proving the feasibility of the detection using the Eddy Current technique.

Coenen [9] proposed a quality assurance system for Glare laminates showing the inspection equipment and methodologies required to the defects detection. Vos [10] developed a one-dimensional model for characterizing acoustic reflection (Pulse-Echo) responses from thin, Glare-3/2 laminates and aluminium. The reflection response showed that the in-plane position and the depth of delaminations can be obtained with a high accuracy. Rosalie et al. [11] introduced an approach using embedded piezo devices made of lead zirconate titanate (PZT), for the detection of delamination in Glare. Bisle et al. [12] showed that a Pulse-Echo inspection provides a very clear and detailed image of the inspected Glare area, when it was subjected to accidental damages like impacts, lightning strike, etc.. Seyed Yaghoubi et al. [13] studied the impact response and damage induced by a drop-weight instrument on Glare 5 fibre-metal laminates of different thicknesses. The damage characteristics were evaluated using both non-destructive ultrasonic and cross-sectional optical pictures. More recently, Fraguas Garcia [14] investigated the effects that the frequency of phased array ultrasonic transducers had on the detection and characterization of delaminations in Glare laminates of different thicknesses.

Meola et al. [15] focused on the aid provided by Infrared Thermography for nondestructive evaluation of Glare. Several specimens made of full composites, hybrid composites, sandwiches, and metals were considered, addressing three kinds of most common damages (delamination, impact damage, fatigue failure). Also, Ibarra-Castanedo 
et al. [16] investigated the use of Infrared Thermography for Glare material inspection. The experimental results demonstrated the possibility to use Thermography for the detection of delamination-type defects and to evaluate the impact severity on Glare.

Among the defects that can occur during Glare manufacturing, the presence of intra-ply gaps and overlaps represent issues that are common with the production of fully composite materials. A gap can hence be defined as a very narrow strip in the panel where pre-preg or at least fibres are missing. At the same position, the thickness may deviate slightly. On the contrary, in an overlap more fibres are present, showing a waviness pattern along with a slightly thicker laminate.

The effect of gaps and overlaps represent a source of flaws and defects (e.g. delaminations), affecting the shape (thickness) and the mechanical properties of the final Glare component: tension strength, compression strength, in-plane strength, fatigue life, etc.

In the past $[17,18]$, some investigations have been performed addressing the effects of gaps and overlaps on the mechanical properties of carbon-epoxy components. More recent results [18] show the performance of gap and overlap configurations in composite materials, providing the conclusions that the ultimate strength is less affected by the different defect configurations at the lamina level (fibre tension, fibre compression, and in-plane shear) as opposed to the laminate level (open hole tension and open hole compression).

The understanding of the effect of gaps and overlaps in components made from FMLs remains an open subject, whose evaluation can be beneficial for the optimization of manufacturing materials, procedures, and ultimately costs. Therefore, the study of the critical parameters related to the presence of gaps and overlaps in Glare laminates represents a compelling demand, especially for the development of an automated tape layering in manufacturing of FMLs, since narrower tapes could introduce a lot more gaps/overlaps.

In this research, the non-destructive detection and evaluation of gaps and overlap in Glare laminates is investigated.

Since it represents a preliminary investigation, only isolated gap and overlap for each laminate has been considered.

First, a defect-manufacturing phase has been realized in order to consider the influence of different gap and overlap widths on Glare specimens.

Then, an ultrasonic non-destructive procedure has been developed in order to evaluate the quality of the laminates in presence of gaps and overlaps.

Finally, an optical evaluation of specimens cut-outs has been performed in order to recognize the effects of gaps and overlaps on the final cured materials.

Results show the reliability of the C-Scan inspection for the gaps and overlaps detection, and the correlation with the cut-outs images in terms of the defects onset, paving the way for the understanding of the effect of gaps and overlaps in Glare laminates.

\section{Gaps and Overlaps Manufacturing}

Specimens of Glare have been manufactured for the gaps and overlaps tests campaign. A Glare 3 grade $\left(0^{\circ} / 90^{\circ}\right)$ has been considered for facilitate the manufacturing operations. A 2/1 lay-up has been selected in order to start the investigation from a configuration that would represent the worst condition for further mechanical properties evaluation. Both $0.3 \mathrm{~mm}$ and 

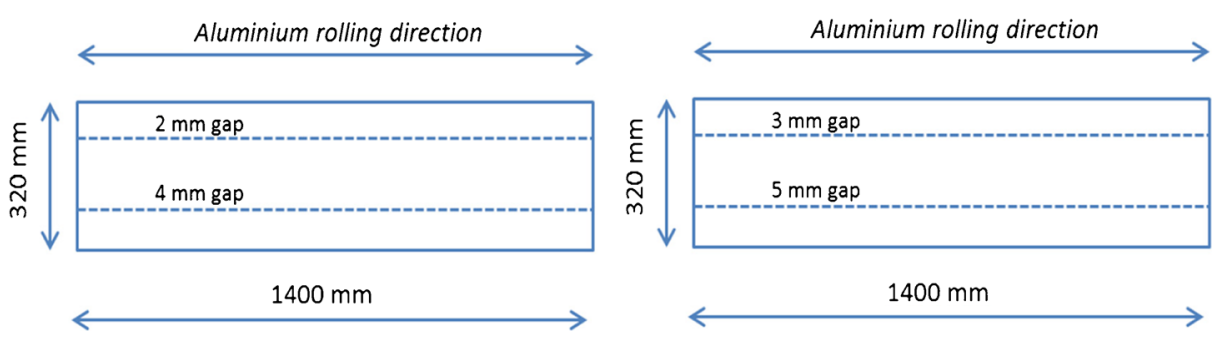

Fig. 2 Sketches of the manufactured Glare 3-2/1-0.3\&0.4 with gaps

$0.4 \mathrm{~mm}$ aluminium thickness for the laminates have been considered in order to evaluate the influence of a different thickness, and hence stiffness, on the gap and overlaps features.

In the first batch 4 laminates with gaps of $2,3,4$, and $5 \mathrm{~mm}$ have been realized in the $0^{\circ}$ prepreg ply, as depicted in Fig. 2. Both aluminium sheet thickness of 0.3 and $0.4 \mathrm{~mm}$ have been considered. The details of the geometry of the produced laminates with gaps are reported in Table 2.

In the second batch of 4 laminates, overlaps of 2,3,4, and $5 \mathrm{~mm}$ have been produced in the $90^{\circ}$ pre-preg ply, as shown in Fig. 3. Both aluminium sheet thickness of 0.3 and $0.4 \mathrm{~mm}$ have been considered. A different rolling direction of the overlaps, with respect to the gaps rolling direction, has been designed since this orientation would be the most likely one to occur during the manufacturing of such laminates. The details of the geometry of the produced laminates with overlaps can be found in Table 3 .

Table 2 Overview of the first batch of Glare laminates specimens for the gaps investigation

\begin{tabular}{llll}
\hline Laminates dimension $[\mathrm{mm}]$ & Laminates ID & Glare features & Gaps width [mm] \\
\hline $1400 \times 320$ & G_03_2 & $3-2 / 1-0.3$ & 2 \\
& G_03_3 & & 3 \\
& G_03_4 & & 4 \\
G_03_5 & $3-2 / 1-0.4$ & 5 \\
G_04_2 & & 2 \\
& G_04_3 & & 3 \\
& G_04_4 & & 4 \\
\hline
\end{tabular}
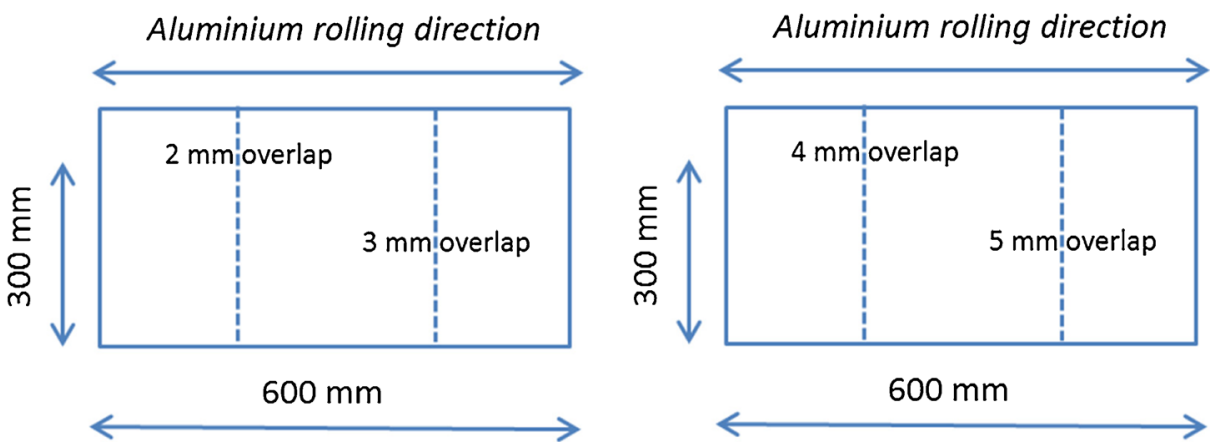

Fig. 3 Sketches of the manufactured Glare 3-2/1-0.3\&0.4 with overlaps 
Table 3 Overview of the second batch of Glare laminates specimens for the overlaps investigation

\begin{tabular}{|c|c|c|c|}
\hline Laminates dimension $[\mathrm{mm}]$ & Laminates ID & Glare features & Overlaps width [mm] \\
\hline \multirow[t]{8}{*}{$600 \times 300$} & O_03_2 & $3-2 / 1-0.3$ & 2 \\
\hline & O_03_3 & & 3 \\
\hline & $\mathrm{O}_{-}^{-} 03-4$ & & 4 \\
\hline & O_03_5 & & 5 \\
\hline & O_04_2 & $3-2 / 1-0.4$ & 2 \\
\hline & O_04_3 & & 3 \\
\hline & $\mathrm{O} 044$ & & 4 \\
\hline & O_04_5 & & 5 \\
\hline
\end{tabular}

The appearance of the cured laminates reveals that the presence of the gaps can be visualized by light contrast based on the local thickness laminate variation, especially for the greater ones, i.e. 4 and $5 \mathrm{~mm}$ gaps (Fig. 4). On the contrary, the presence of the overlaps is more difficult to evaluate (Fig. 5). However, the detection of gaps and overlaps was efficient mainly because of the size of the considered panels, which allows an easy visual light-based inspection. If wider laminates are considered, a more effective inspection is needed.

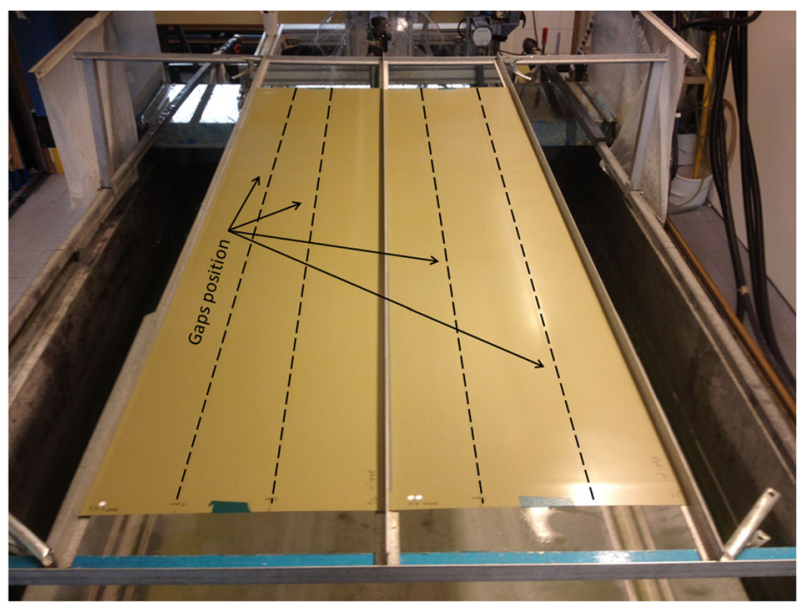

Fig. 4 Glare laminates with gaps

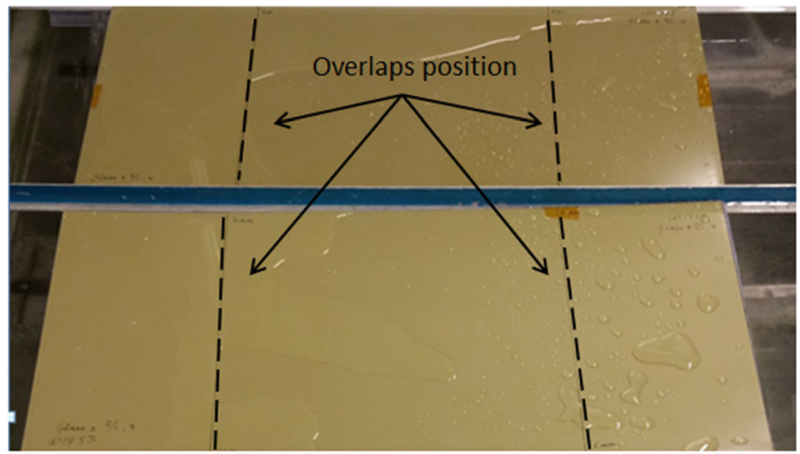

Fig. 5 Glare laminates with overlaps 
Table 4 Data of C-Scan runs for the gaps investigation

\begin{tabular}{ll}
\hline Width of the ultrasound beam $[\mathrm{mm}]$ & 4 \\
\hline Scanning pattern resolution $[\mathrm{mm}]$ & 1 \\
Scanning rate $[\mathrm{mm} / \mathrm{s}]$ & 200 \\
Transducer distance $[\mathrm{cm}]$ & 20 \\
Transducer frequency $[\mathrm{MHz}]$ & 10 \\
Rejection level of attenuation $[\mathrm{dB}]$ & -6 \\
\hline
\end{tabular}

\section{Ultrasonic Non-Destructive Evaluation of Gaps and Overlaps}

The manufactured Glare laminates with gaps and overlaps have been subjected to an ultrasonic non-destructive analysis. This has been performed by the C-Scan facility available at the Delft Aerospace Structures and Materials Laboratory (DASML).

A C-scan system, which is routinely used in the aerospace industry, is based on sending ultrasound signal through the laminate where its attenuation is measured at different positions over its surface. Usually, uniform scanning patterns over the laminate are used, with lines at a small distance from each other. The scanning pattern resolution represents the space between these scanning lines. The measured attenuation is displayed as a greyscale or colour image [9].

\subsection{Gaps NDE}

For this investigation, the diameter of the ultrasound beam created by the transducer has been set equal to $4 \mathrm{~mm}$, and a resolution of $1 \mathrm{~mm}$ (one pulse $/ \mathrm{mm}$ ) has been used for the scanning pattern. The scanning rate has been set equal to $200 \mathrm{~mm} / \mathrm{s}$. The water squirters (water is used as medium) have been placed at a distance of $20 \mathrm{~cm}$, with the laminates placed in the middle. The transducer frequency was set at $10 \mathrm{MHz}$, since this represents the most sensitive frequency for general Glare quality assessment [9]. During the analysis of the acquired data $\mathrm{a}-6 \mathrm{~dB}$ rejection level of the attenuation is used with respect to a reference flawless area. So, all areas with attenuation larger than $6 \mathrm{~dB}$ will be indicated. Table 4 summarizes the adopted C-scan setup.

Figure 6 shows the results of the C-Scan analysis performed on the Glare specimens with aluminium thickness of $0.3 \mathrm{~mm}$ with 5 and $3 \mathrm{~mm}$ gaps (top) and 4 and $2 \mathrm{~mm}$ gaps (bottom).

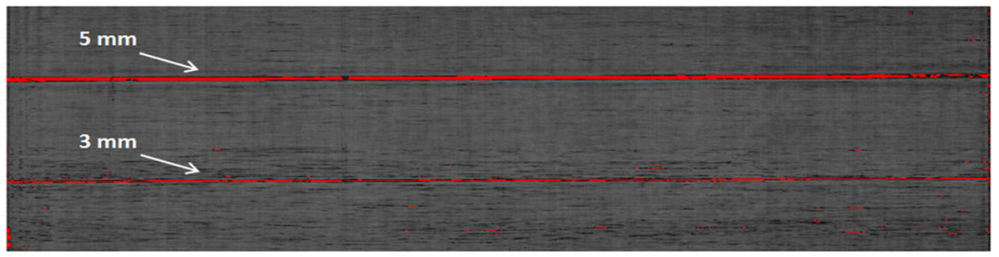

a

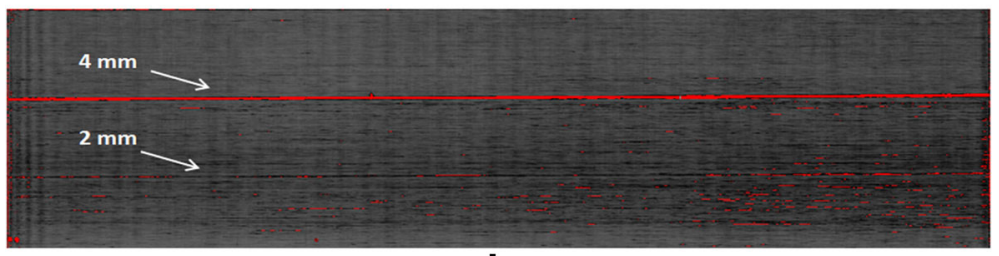

b

Fig. 6 C-Scan of the Glare 3-2/1-0.3 laminates with gaps; 5 and $3 \mathrm{~mm}$ gaps (a), 4 and 2 mm gaps (b) 


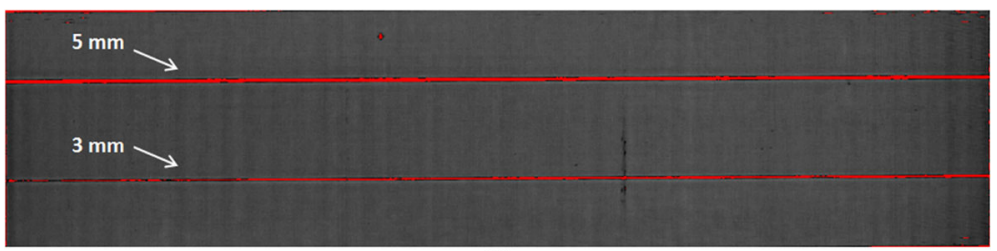

a

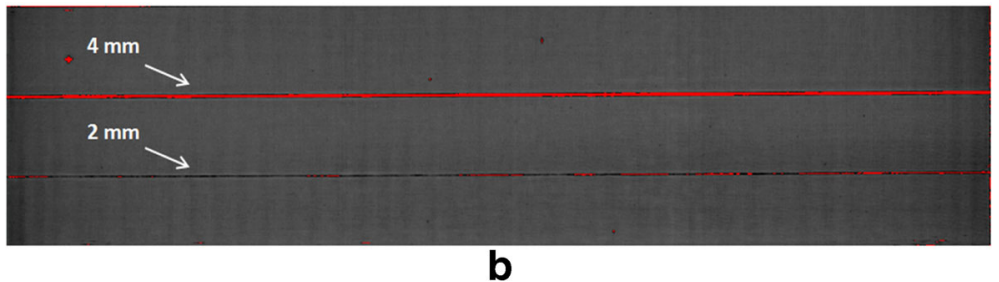

Fig. 7 C-Scan of the Glare 3-2/1-0.4 laminates with gaps; 5 and $3 \mathrm{~mm}$ gaps (a), 4 and 2 mm gaps (b)

Qualitatively, it can be seen from Fig. 6 that for gaps of 5, 4, and $3 \mathrm{~mm}$, the C-scan provides an indication of the presence of the defect due to the attenuation of the gap area (red line) with respect to the undisturbed material. On the other hand, the presence of the $2 \mathrm{~mm}$ gaps is not clearly detected. Such occurrence can be attributed to the expected gap reduction due to the resin flow from the adjacent pre-preg, but more likely from an inaccurate selection of the Cscan parameters for the current defect investigation, which will be adapted as follow.

In order to improve the C-scan routine, the scanning rate has been slowed down to $100 \mathrm{~mm} /$ $\mathrm{s}$, the transducers distance has been reduced to $15 \mathrm{~cm}$, and the laminates have been tilted to a few degrees $\left(2^{\circ}-3^{\circ}\right)$ with respect to the transducers motion, in order to misalign the orientation of the gaps with the orientation of the ultrasound scanning pattern. The adopted changes were intended to reduce the interference of the water drops with the water jet of the bottom squirter, which would lead to an intermittent interruption of the ultrasonic signal transmission [9].

Figure 7 shows the results of the C-Scan analysis performed on the Glare specimens with aluminium thickness of $0.4 \mathrm{~mm}$ with 5 and $3 \mathrm{~mm}$ gaps (top) and 4 and $2 \mathrm{~mm}$ gaps (bottom).

The obtained results show the reduction of the scatter for a better evaluation of all the four different gap widths, proving the benefits of the adopted changes of the $\mathrm{C}$-scan routine. Moreover, the tendency of the pre-preg from the adjacent material to fill the gap due to the autoclave pressure can also be deduced by a non-homogeneous width of the gap lines, significantly shown in the $2 \mathrm{~mm}$ gap area, where a different level of attenuation (red and dark grey) are found.

The effectiveness of the gap width evaluation based on the $\mathrm{C}$-scan is also addressed. Table 5 shows the values of the width of the gaps measured directly from the $\mathrm{C}$-scan output by means

Table 5 Gap width values obtained from the C-Scan inspection

\begin{tabular}{lll}
\hline Specimen & $\begin{array}{l}\text { Gap width [mm] } \\
\text { manufactured }\end{array}$ & $\begin{array}{l}\text { Gap width [mm] } \\
\text { observed (max value) }\end{array}$ \\
\hline Glare 3-2/1-0.3 & 5 & 5 \\
Glare 3-2/1-0.3 & 4 & 4 \\
Glare 3-2/1-0.3 & 3 & 2 \\
Glare 3-2/1-0.3 & 2 & - \\
Glare 3-2/1-0.4 & 5 & 4 \\
Glare 3-2/1-0.4 & 4 & 3 \\
Glare 3-2/1-0.4 & 3 & 2 \\
Glare 3-2/1-0.4 & 2 & 2 \\
\hline
\end{tabular}


Table 6 Average attenuation levels of the Glare laminates; reference and with gaps

\begin{tabular}{|c|c|c|c|c|c|c|c|c|c|c|}
\hline \multirow{2}{*}{$\begin{array}{l}\text { Specimen } \\
\text { Flaw }\end{array}$} & \multicolumn{5}{|c|}{ Glare 3-2/1-0.3 } & \multicolumn{5}{|c|}{ Glare 3-2/1-0.4 } \\
\hline & Gap & & & & & Gap & & & & \\
\hline Width [mm] & Reference & 5 & 4 & 3 & 2 & Reference & 5 & 4 & 3 & 2 \\
\hline Average attenuation level [dB] & 37.50 & 83.83 & 73.00 & 59.66 & / & 28.33 & 75.83 & 70.16 & 45.83 & 38.83 \\
\hline Standard deviation $[\mathrm{dB}]$ & 2.16 & 20.73 & 9.05 & 14.43 & / & 0.51 & 9.72 & 11.56 & 9.66 & 3.65 \\
\hline
\end{tabular}

of a dedicated software (Zeus3). What shows, is that there is a good estimation of the different gap widths, though confirming the reduction of the gap widths due to the curing cycle.

Finally, the average attenuation values of the reference material with respect to the gap areas is measured and reported in Table 6 and Fig. 8. The average values are computed considering six measurement points selected along the length of the laminates. Results show that for both laminates made of aluminium thicknesses of 0.3 and $0.4 \mathrm{~mm}$, the attenuation values are higher for wider gaps. These results are justified by the thickness variations locally induced by the

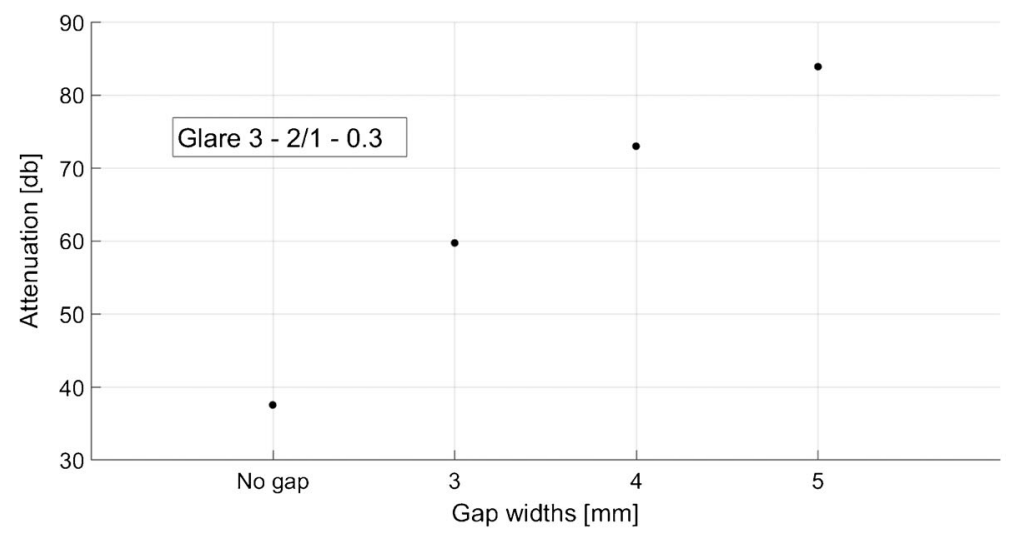

a

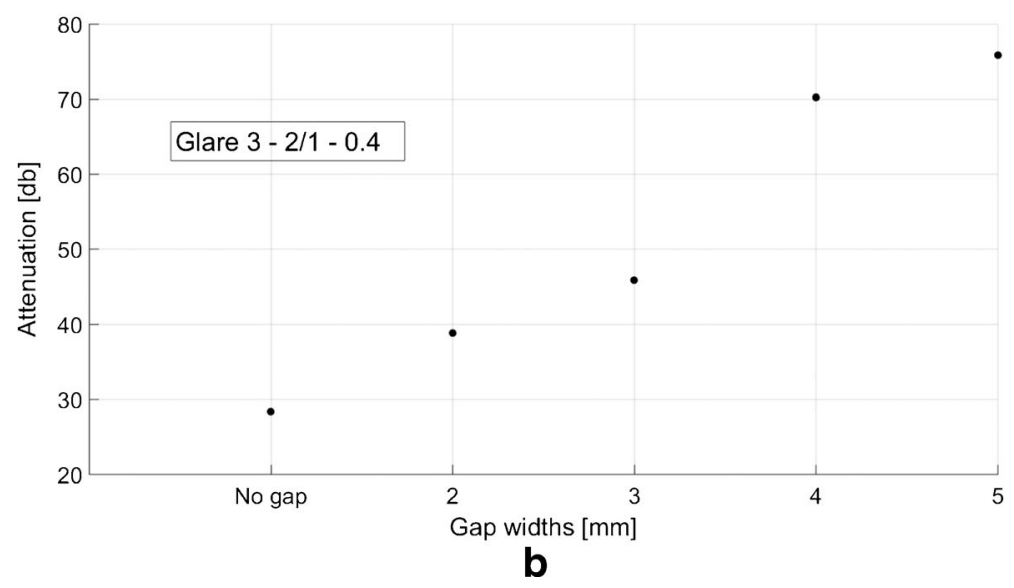

Fig. 8 Variation of the attenuation values due to the gaps presence: $0.3 \mathrm{~mm}$ aluminium thickness (a), $0.4 \mathrm{~mm}$ aluminium thickness (b) 
presence of the gap, as it will be presented later (see Table 8). A higher gap width (5 mm) yields to a greater local thickness step compared to a lower gap width $(2 \mathrm{~mm})$, resulting in a greater attenuation. Therefore, the severity of the gap presence, related to the gap width, can be effectively related to the attenuation level. Moreover, it can be seen that the modified C-scan routine adopted for the laminates with $0.4 \mathrm{~mm}$ aluminium thick lead to an overall reduced value of the standard deviations, proving its effectiveness for assessing the severity of the gaps.

\subsection{Overlaps NDE}

The adapted C-scan setup used for the gap investigation of Glare 3-2/1-0.4 has been used for the analysis of the Glare laminates with overlaps.

Figure 9 shows the C-Scan of the Glare specimens for the laminates with aluminium thickness of $0.3 \mathrm{~mm}$ with 5 and $4 \mathrm{~mm}$ overlaps (Fig. 9a) and 3 and $2 \mathrm{~mm}$ overlaps (Fig. 9b).

Likewise, Fig. 10 shows the C-Scan of the Glare specimens for the laminates with aluminium thickness of $0.4 \mathrm{~mm}$ with 5 and $4 \mathrm{~mm}$ overlaps (Fig. 11a) and 3 and $2 \mathrm{~mm}$ overlaps (Fig. 11b).

Figures 9 and 10 indicate the presence of a darker area in correspondence of the presence of the manufactured overlaps. However, differently from the gaps investigation, the areas for overlap widths measurement were not possible to clearly define. Therefore, the overlaps width estimation is performed in the next section by means of optical evaluation.

In addition, from the values reported in Table 7, it can be seen that the average attenuation levels are within the $6 \mathrm{~dB}$ with respect to the reference (flawless) area, except for the overlap of $5 \mathrm{~mm}$ in the laminate with $0.3 \mathrm{~mm}$ aluminium thick, where the threshold is exceed by $1 \mathrm{~dB}$ only. Therefore, differently from the gaps

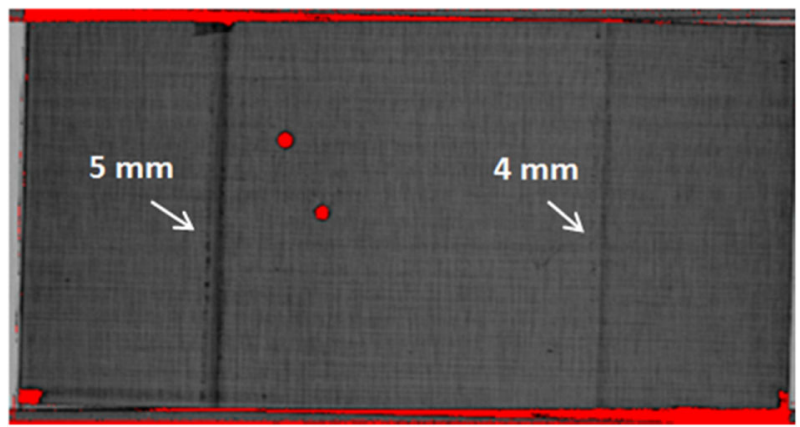

a

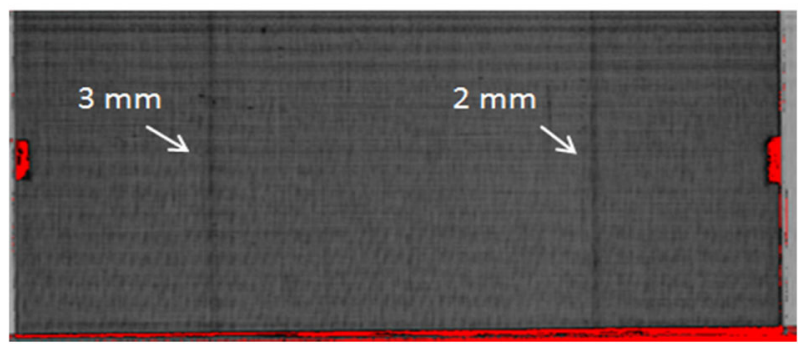

b

Fig. 9 C-Scan of the Glare 3-2/1-0.3 laminates with overlaps; 5 and 4 mm overlaps (a), 4 and 2 mm overlaps (b) 


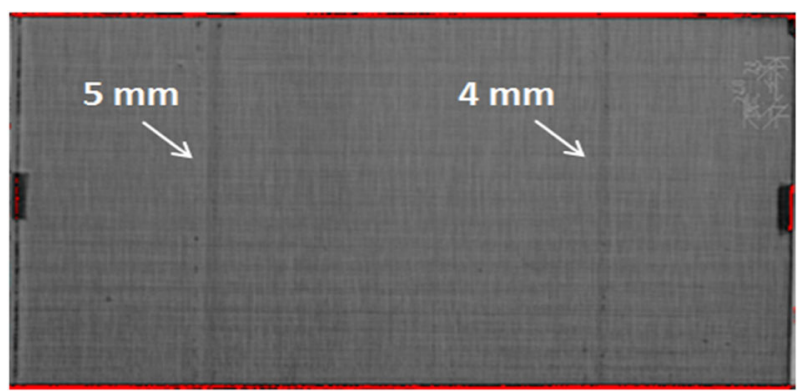

a

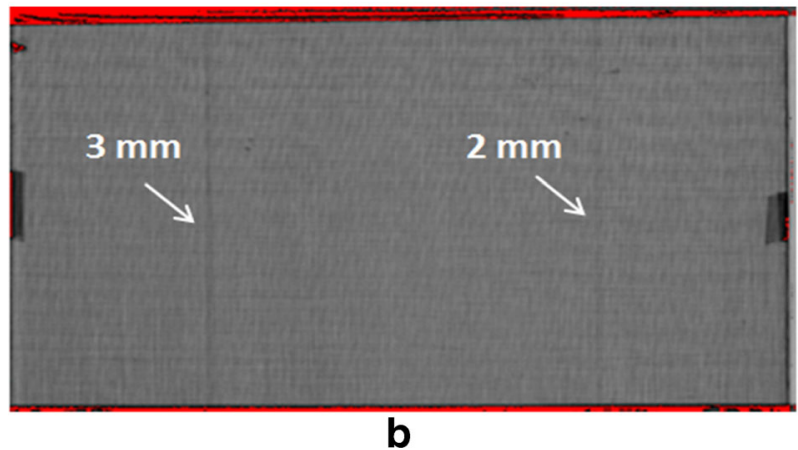

Fig. 10 C-Scan of the Glare 3-2/1-0.4 laminates with overlaps; 5 and 4 mm overlaps (a), 4 and 2 mm overlaps (b)

Fig. 11 Cross sections of a single specimen arranged for the optical inspection

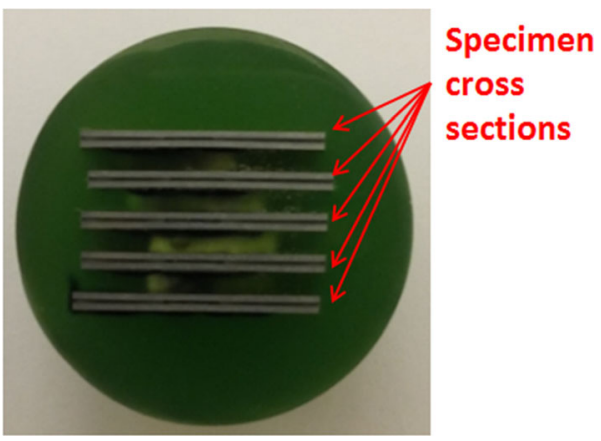

Table 7 Average attenuation levels of the Glare laminates; Reference and with overlaps

\begin{tabular}{|c|c|c|c|c|c|c|c|c|c|c|}
\hline \multirow{2}{*}{$\begin{array}{l}\text { Specimen } \\
\text { Flaw }\end{array}$} & \multicolumn{5}{|c|}{ Glare 3-2/1-0.3 } & \multicolumn{5}{|c|}{ Glare 3-2/1-0.4 } \\
\hline & Overlap & & & & & Overlap & & & & \\
\hline Width [mm] & Reference & $5 \mathrm{~mm}$ & $4 \mathrm{~mm}$ & $3 \mathrm{~mm}$ & $2 \mathrm{~mm}$ & Reference & $5 \mathrm{~mm}$ & $4 \mathrm{~mm}$ & $3 \mathrm{~mm}$ & $2 \mathrm{~mm}$ \\
\hline $\begin{array}{l}\text { Average attenuation } \\
\text { level }[\mathrm{dB}]\end{array}$ & 52.00 & 59.33 & 54.33 & 53.33 & 53.67 & 44.33 & 45.67 & 46.33 & 44.33 & 41.67 \\
\hline Standard deviation $[\mathrm{dB}]$ & 1.00 & 1.53 & 0.58 & 1.53 & 3.21 & 1.53 & 0.58 & 3.22 & 2.08 & 1.53 \\
\hline
\end{tabular}


investigation, the overlaps regions can be considered as flawless material based on the attenuation threshold level. However, the overlap may introduce small thickness variations which might affect the assembly with back-up structures, e.g. stringers and doublers components, and the different effect with respect to the different aluminium thicknesses $(0.3$ and $0.4 \mathrm{~mm})$ has to be considered. Conversely from gaps, however, the thickness variations in overlaps is difficult to accurately evaluate, since the area affected by overlaps does not present a clear peak, as it will be presented in section 4.

\section{Optical evaluation of cut-outs}

The presence of gaps and overlaps is effectively detected by means of a conventional C-scan analysis. Results reveal that gaps produce evident signal attenuation with respect to a flawless laminate area, while the presence of overlaps does not produce any significant attenuation. The absence of pre-preg and fibre related to the gap presence might cause porosity due to the eccentricity at the gap location and will affect the Glare mechanical properties. Overlaps instead will not introduce any defect in the Glare materials, but can locally affect the laminate thickness, which in turn can cause problems for a later assembly to back-up structures. In order to interpret the results obtained from the C-scan evaluation, a microscopic assessment is necessary. Therefore, in this section, the presence of gaps and overlaps in the previously manufactured Glare laminates is investigated by evaluation of cross section cut-outs. The images show the critical features caused by gaps and overlaps, which are represented by the out-of-ply fibre waviness, mainly due to the pressure that forces the adjacent pristine ply onto the defect, and the ply thickness variations. $\$.

\subsection{Gaps optical evaluation}

For each specimen, five small pieces (about $2 \mathrm{~cm}$ of width) have been cut out along the length of each specimen in correspondence to the gaps position. To be analysed by a microscope, the samples have been placed in a resin and a proper inspection surface was created by abrasion, as shown in Fig. 11. For practical reasons, only gaps of 2 and $4 \mathrm{~mm}$ have been addressed, for both 0.3 and $0.4 \mathrm{~mm}$ aluminium thicknesses specimens.

The microscopic evaluation of a cross section for a $2 \mathrm{~mm}$ gap is shown in Fig. 12. The images clearly show the presence of the gap area, which is filled by the epoxy resin without fibres. The

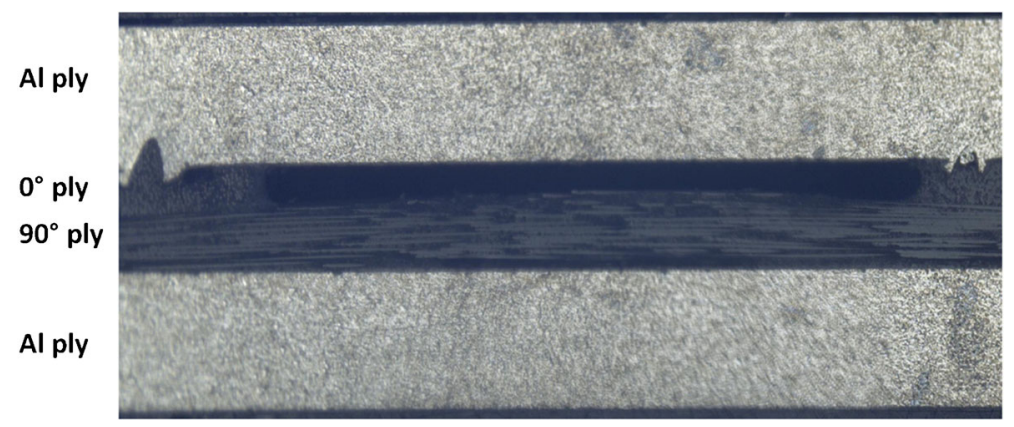

Fig. 12 Detail of the gap presence in Glare 3-2/1-0.3 

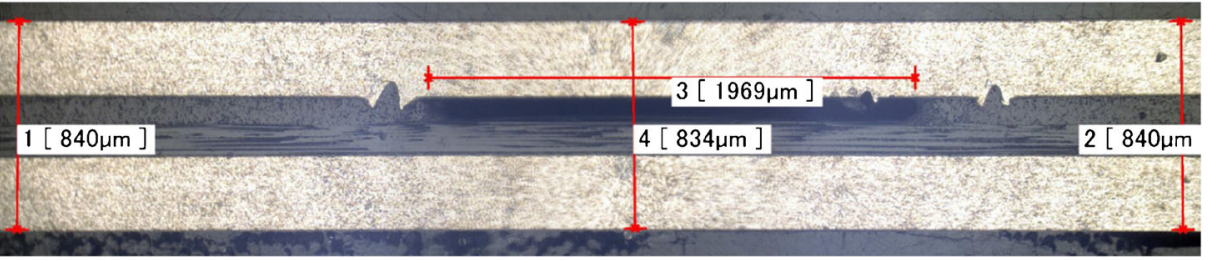

Fig. 13 Cut-out of the Glare specimen of $0.3 \mathrm{~mm}$ aluminium thickness with a manufactured gap of $2.6 \mathrm{~mm}$

scratches (marks) visible in the upper aluminium layer (left side) have been produced by the cutter during the process of gap manufacturing. Such marks provided information concerning the manufactured gap width (MGW). From the measurement of the distance between the two marks, we found out that the MGW at the start of the autoclave process was $2.6 \mathrm{~mm}$ for both 0.3 and $0.4 \mathrm{~mm}$ aluminium thickness specimens. Therefore, from now a gap width of $2.6 \mathrm{~mm}$ will be addressed in order to evaluate the reduction of the gap width due to the cure process. The same width check for the $4 \mathrm{~mm}$ gaps based on the marks distance resulted instead to confirm on average the intended gap width realized during the manufacturing stage.

In addition, four parameters have been considered for a quantitative optical evaluation of the effect of a gap: (1) the actual gap width (AGW) after the cure process, (2) the laminate thickness in the gap area, and the laminate thicknesses (3-4) in the areas not affected by the gap presence, respectively at the left and at the right of the gap. The microscope cross-section images of the specimens for both the aluminium thicknesses with the initial gap width of $2.6 \mathrm{~mm}$ are reported in Figs. 13 and 14, respectively.

Table 8 presents the average values along with the standard deviations of the mentioned parameters for all of the realized cross sections (5) from the considered specimens.

In order to obtain some general and useful insight/understanding of the geometrical effects of the gap presence, Table 9 shows the average reductions of both the MGW and the laminate thickness. From a comparison of the results presented in Tables 8 and 9 it can be seen that since a thicker aluminium layer $(0.4 \mathrm{~mm})$ has a better resistance towards the autoclave pressure, the thickness variations for the thinner metal layer $(0.3 \mathrm{~mm})$ are larger, see Fig. 15 . On the other hand, it can be observed that, for the same gap width, the average MGW reduction is higher in the aluminium $0.4 \mathrm{~mm}$ thick. This result can also be related to the different stiffness: when the stiffness is higher, the outer metal layer remains relatively flatter and a more uniformly pressure is acting on the pre-preg in the gap areas. However, more tests (or numerical simulations) need to validate this assumption, which is based on average values only.

Last, it can be seen that for the laminate with the same aluminium thickness, the average thickness reduction is larger for a gap $4 \mathrm{~mm}$ wide compared to a gap $2.6 \mathrm{~mm}$ wide. This result

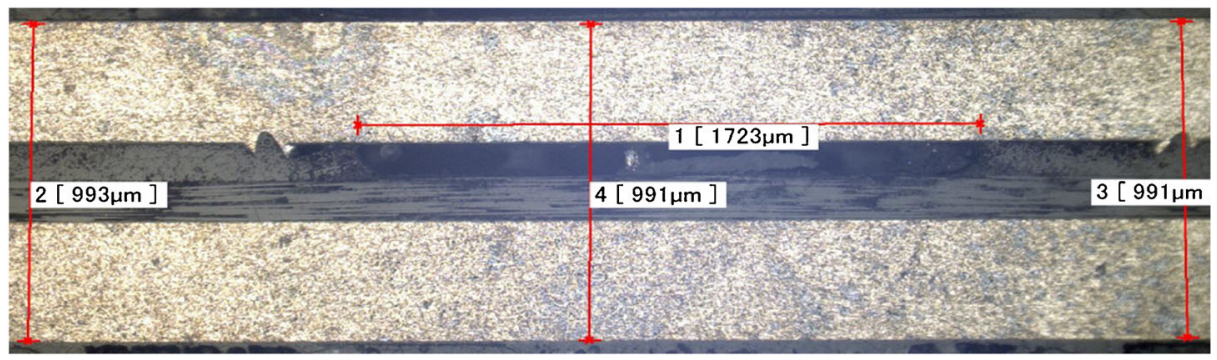

Fig. 14 Cut-out of the Glare specimen of $0.4 \mathrm{~mm}$ aluminium thickness with a manufactured gap of $2.6 \mathrm{~mm}$ 
Table 8 Values of the geometric parameters evaluated for the gaps investigation; STD = Standard deviation

\begin{tabular}{|c|c|c|c|c|c|c|c|c|}
\hline \multirow{2}{*}{$\begin{array}{l}\text { Specimen } \\
\text { Glare } 3-2 / 1-0.3\end{array}$} & \multirow{2}{*}{$\begin{array}{l}\text { Gap width } \\
\text { manufactured }[\mathrm{mm}] \\
2.6\end{array}$} & \multirow{2}{*}{$\begin{array}{l}\text { Gap width } \\
\text { actual }[\mathrm{mm}] \\
1.79\end{array}$} & \multicolumn{2}{|c|}{$\begin{array}{l}\text { Laminate } \\
\text { thickness left } \\
{[\mathrm{mm}]}\end{array}$} & \multicolumn{2}{|c|}{$\begin{array}{l}\text { Laminate } \\
\text { thickness gap } \\
\text { area }[\mathrm{mm}]\end{array}$} & \multicolumn{2}{|c|}{$\begin{array}{l}\text { Laminate } \\
\text { thickness right } \\
{[\mathrm{mm}]}\end{array}$} \\
\hline & & & Average & STD & Average & STD & Average & STD \\
\hline & & 1.94 & 0.83 & 0.090 & 0.82 & 0.006 & 0.84 & 0.005 \\
\hline & & 1.97 & & & & & & \\
\hline & & 1.42 & & & & & & \\
\hline & & 2.35 & & & & & & \\
\hline & 4.0 & 3.46 & Average & STD & Average & STD & Average & STD \\
\hline & & 3.37 & 0.81 & 0.007 & 0.79 & 0.010 & 0.81 & 0.006 \\
\hline & & 3.76 & & & & & & \\
\hline & & 3.74 & & & & & & \\
\hline & & 3.57 & & & & & & \\
\hline \multirow[t]{10}{*}{ Glare $3-2 / 1-0.4$} & 2.6 & 1.35 & Average & STD & Average & STD & Average & STD \\
\hline & & 1.32 & 0.98 & 0.008 & 0.98 & 0.008 & 0.98 & 0.006 \\
\hline & & 1.72 & & & & & & \\
\hline & & 1.81 & & & & & & \\
\hline & & 1.92 & & & & & & \\
\hline & 4.0 & 2.91 & Average & STD & Average & STD & Average & STD \\
\hline & & 3.33 & 0.98 & 0.007 & 0.98 & 0.009 & 0.99 & 0.011 \\
\hline & & 3.55 & & & & & & \\
\hline & & 3.59 & & & & & & \\
\hline & & 3.51 & & & & & & \\
\hline
\end{tabular}

Table 9 Gaps width obtained from the cross section cut-outs optical inspection

\begin{tabular}{lllll}
\hline Specimen & $\begin{array}{l}\text { Gap width } \\
\text { manufactured [mm] }\end{array}$ & $\begin{array}{l}\text { Average gap } \\
\text { width actual [mm] }\end{array}$ & $\begin{array}{l}\text { Average gap width } \\
\text { reduction [\%] }\end{array}$ & $\begin{array}{l}\text { Average laminate } \\
\text { thickness reduction [\%] }\end{array}$ \\
\hline Glare 3-2/1-0.3 & 2.6 & 1.90 & 27 & 1.6 \\
& 4.0 & 3.60 & 10 & 2.4 \\
Glare 3-2/1-0.4 & 2.6 & 1.60 & 38 & 0.2 \\
& 4.0 & 3.40 & 15 & 0.8 \\
\hline
\end{tabular}

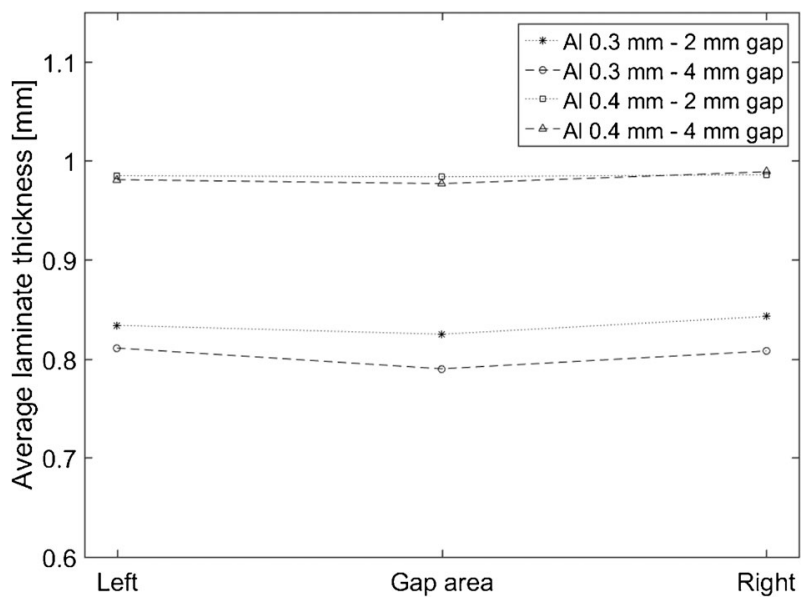

Fig. 15 Trend of the average laminate thickness due to the presence of the gap 


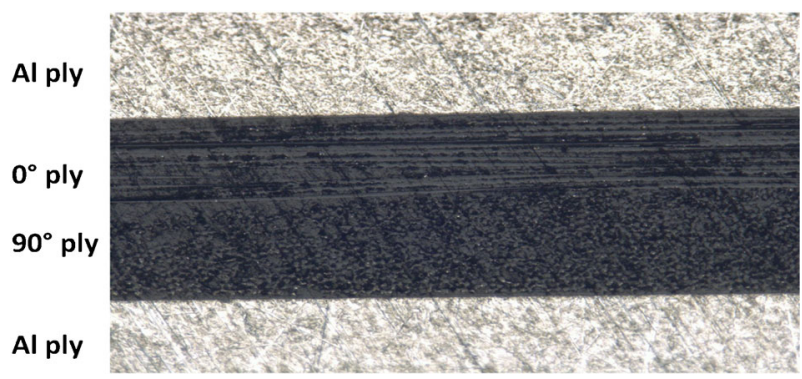

Fig. 16 Overlap in Glare 3-2/1-0.3

is in agreement with the variation of the attenuation level reported in Table 6, and confirms the influence of the laminate thickness on the ultrasonic signal attenuation.

\subsection{Overlaps optical evaluation}

As for the gap investigation, the laminates with overlaps have been analysed using cross section cutouts at several overlap positions. For each overlap configuration, four cross sections were made, and were prepared for microscopic evaluation, as previously shown in Fig. 11. For practical reasons, only gaps of 3 and $4 \mathrm{~mm}$ have been addressed, for both 0.3 and $0.4 \mathrm{~mm}$ aluminium thicknesses specimens.

As expected from the C-scan analysis, the overlaps attenuation levels stay within the $6 \mathrm{~dB}$ threshold with respect to the reference pristine material. Whether this aspect is sufficient for considering the laminate not affected by overlaps it will be addressed in future experimental testing. In Fig. 16 the waviness in the fibres at the overlap is shown, and proving the absence of defects. Differently, from the gaps, the presence of the overlaps does not significantly differ between laminates with different aluminium thickness $(0.3$ and $0.4 \mathrm{~mm})$. Therefore, only the results from $0.3 \mathrm{~mm}$ aluminium are reported.

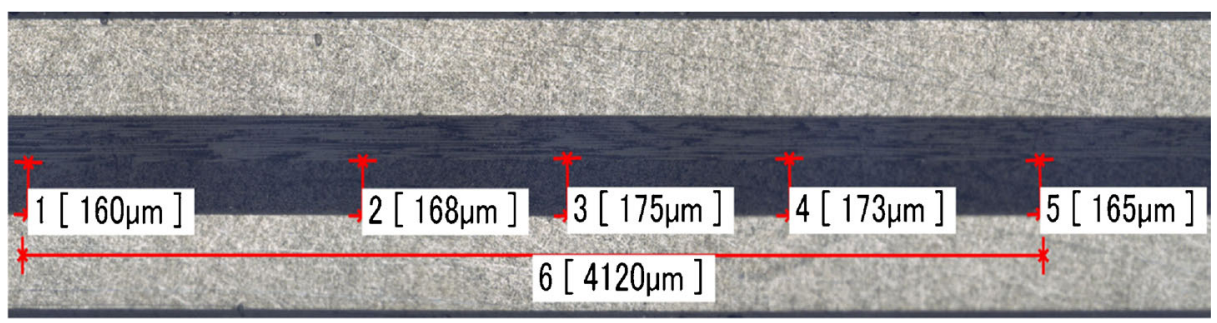

Fig. $1790^{\circ}$ ply thickness variation due to $3 \mathrm{~mm}$ overlap (Glare 3-2/1-0.3)

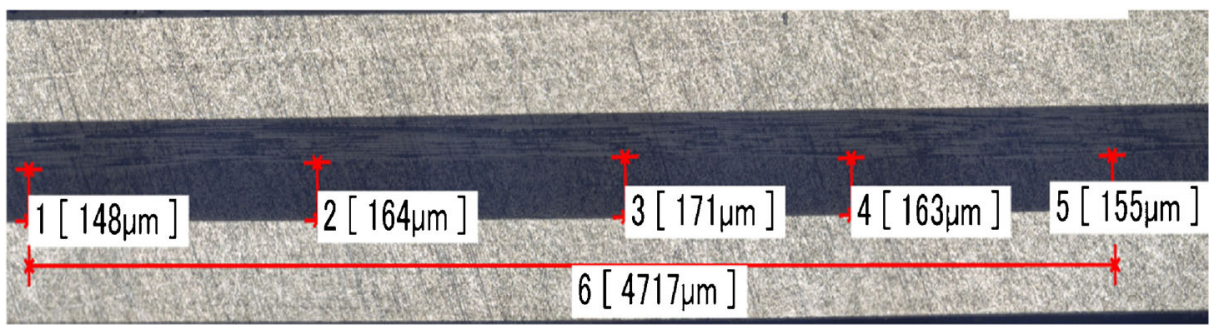

Fig. $1890^{\circ}$ ply thickness variation due to $4 \mathrm{~mm}$ overlap (Glare $32 / 1-0.3$ ) 
Table 10 Thickness variation of the $90^{\circ}$ ply affected by the overlap

\begin{tabular}{lllll}
\hline Specimen & $\begin{array}{l}\text { Overlap } \\
\text { width [mm] }\end{array}$ & $\begin{array}{l}90^{\circ} \text { reference } \\
\text { thickness-left-[mm] }\end{array}$ & $\begin{array}{l}90^{\circ} \text { Overlap max } \\
\text { thickness [mm] }\end{array}$ & $\begin{array}{l}90^{\circ} \text { reference } \\
\text { thickness-right-[mm] }\end{array}$ \\
\hline Glare 3-2/1-0.3 & 3 & 160 & 175 & 165 \\
& 4 & 148 & 171 & 155 \\
\hline
\end{tabular}

The overlaps, however, change the thickness of the laminate especially if multiple overlaps occur in the same region of a laminate with a higher number of plies, which can be detrimental to the compression properties, and even if not really detrimental from a mechanical point of view, they are unacceptable for the customers.

Figures 17 and 18, show the thickness variations of the $90^{\circ}$ ply in overlaps of 3 and $4 \mathrm{~mm}$, respectively. The measurements indicate an extension of the material area affected by the overlap presence and the associated thickness increment, as also reported in Table 10.

\section{Conclusions}

An experimental study was performed on the detectability and geometrical visibility of gaps and overlaps of different widths in Glare laminates.

The detection has been obtained by means of a C-scan ultrasonic routine which showed the gap width reduction after the cure of the laminates, and the attenuation level in the gap areas with respect to the reference (healthy) area which result to be higher for wider gaps, enabling the estimation of the gaps severity along with their position. The $\mathrm{C}$-scan routine performed for overlaps, instead, led to ultrasonic attenuation levels which were slightly lower than the reference area, foreseeing the conclusion that the effect of the presence of the overlaps leads to a potential different impact with respect to the presence of the gaps in terms of defect onset and mechanical properties reduction.

Finally, several cross section images were analysed with a microscope. The images of gaps showed a general width reduction with respect to the initial manufactured gap. The gap is completely filled with the epoxy from the contiguous pre-preg, and some of the fibres shift a little into the gap. Conversely, the cross sectional images from the overlaps, do not show any sign of defect onset, validating the results of the $\mathrm{C}$-scan analysis. An overall laminate thickness increase along with some fibre waviness are observed, indicating a possible impact on the compressive strength and for the assembly with stringers or doublers.

Future works can investigate the presence of gaps and overlaps in different Glare grades, aiming to provide a comprehensive description of the effects of gaps and overlaps which result to be essential to understanding failure mechanisms in the vicinity of a gap and overlap flaws.

Acknowledgements This research was carried out under project number T11.6.14523 in the framework of the Research Program of the Materials innovation institute (M2i) (www.m2i.nl) and Fokker Aerostructures.

Open Access This article is distributed under the terms of the Creative Commons Attribution 4.0 International License (http://creativecommons.org/licenses/by/4.0/), which permits unrestricted use, distribution, and reproduction in any medium, provided you give appropriate credit to the original author(s) and the source, provide a link to the Creative Commons license, and indicate if changes were made. 


\section{References}

1. Sinke, J.: Manufacturing of GLARE Parts and Structures. Appl. Compos. Mater. 10, 293-305 (2003)

2. Hagenbeek, M.: Characterisation of Fibre Metal Laminates under Thermo-mechanical Loadings. Ph.D. dissertation, Delft University of Technology (2005)

3. Vlot, A., Gunnink, J.W.: Fibre Metal Laminates an introduction. Kluwer, Dordrecht (2001)

4. Alderliesten, R., Hagenbeek, M., Homan, J.J., Hooijmeijer, P.A., De Vries, T.J., Vermeeren, C.: Fatigue and Damage Tolerance of Glare. Appl. Compos. Mater. 10, 223-242 (2003)

5. Fahr, A., Chapman, C.E., Laliberte, J.F., Forsyth, D.S., Poon, C.: Nondestructive Evaluation Methods for Damage Assessment in Fiber-Metal Laminates. Polym. Compos. 21, 568-575 (2000)

6. Heida, J.H., Platenkamp, D.J.: In-service inspection of Glare fuselage structures. National Aerospace Laboratory NLR-TP-2001-608 Report, The Netherlands (2001)

7. Sinke, J.: Some Inspection Methods for Quality Control and In-service Inspection of GLARE. Appl. Compos. Mater. 10, 277-291 (2003)

8. Heida, J.H., Platenkamp D.J., Bosch, A.F.: Eddy current Inspection of Glare riveted structures. National Aerospace Laboratory NLR report CR-2005-110, The Netherlands (2006)

9. Coenen, R.A.M.: Design of a Quality Assurance System for Structural Laminates. Ph.D. dissertation, Delft University of Technology (1998)

10. Vos, J.: Characterisation of laminated construction materials based on ultrasonic reflection measurements. Ph.D. dissertation, Delft University of Technology (2000)

11. Rosalie, S.C., Vaughan, M., Bremner, A., Chiu, W.K.: Variation in the group velocity of Lamb waves as a tool for the detection of delamination in GLARE aluminium plate-like structures. Compos. Struct. 66, 77-86 (2004)

12. Bisle, W., Meier, T., Mueller, S., Rueckert, S.: In-service Inspection Concept for GLARE - An Example for the Use of New UT Array Inspection Systems. European Conference on Non-Destructive Testing, Tu.2.1.1 (2006). https://pdfs.semanticscholar.org/6701/463fdelbe8e33d8886c75c0aafcdd8a32915.pdf

13. Seyed Yaghoubi, A., Liu, Y., Liaw, B.: Low-velocity Impact on GLARE 5 Fiber-Metal Laminates: Influences of Specimen Thickness and Impactor Mass. J. Aerosp. Eng. 25, 409-420 (2012)

14. Fraguas Garcia, V.: Multi-Frequency Approach to Phased Array Ultrasonic Inspection of GLARE Laminates. M.Sc. dissertation, Delft University of Technology (2014)

15. Meola, C., Carlomagno, G.M., Squillace, A., Vitiello, A.: Non-destructive evaluation of aerospace materials with lock-in thermography. Eng. Fail. Anal. 13, 380-388 (2006)

16. Ibarra-Castanedo, C., Avdelidis, N.P., Grinzato, E.G., Bison, P.G., Marinetti, S.: Delamination detection and impact damage assessment of GLARE by active thermography. Int. J. Mater. Prod. Technol. 41, 1-4 (2011)

17. Sawicki, A.J., Minguet, P.L.: The effect of intraply overlaps and gaps upon the compression strength of composite laminates, pp. 744-754. American Institute of Aeronautics and Astronautics, Inc.. 39th Structural Dynamics, and Materials Conference and Exhibit, Long Beach (1998)

18. Croft, K., Lessard, L., Pasini, D., Hojjati, M., Chen, J., Yousefpour, A.: Experimental study of the effect of automated fiber placement induced defects on performance of composite laminates. Compos. Part A. 42, 484-491 (2011) 